OPEN ACCESS

Edited by:

Andrew T. Gewirtz, Georgia State University,

United States

Reviewed by:

Jian-gao Fan,

Shanghai Jiaotong University, China

Qin Pan,

Shanghai Jiaotong University, China

Kristina Marie Feye,

National Center for Toxicological

Research (FDA), United States

${ }^{*}$ Correspondence:

Qing Xie

xieqingrih@163.com

Zhujun Cao

estherlucifer@163.com

Gangde Zhao

296747726@qq.com

${ }^{\dagger}$ These authors share first authorship

Specialty section:

This article was submitted to Microbiome in Health and Disease, a section of the journal

Frontiers in Cellular and Infection Microbiology

Received: 30 June 2021 Accepted: 13 December 2021 Published: 17 January 2022

Citation:

Huang $Y$, Gan $Q$, Lai R, Wang W, Guo S, Sheng Z, Chen L, Guo Q, Cai W, Wang $H$, Zhao G, Cao $Z$ and Xie Q (2022) Application of Fatty Liver Inhibition of Progression Algorithm and Steatosis, Activity, and Fibrosis Score to Assess the Impact of NonAlcoholic Fatty Liver on Untreated Chronic Hepatitis B Patients. Front. Cell. Infect. Microbiol. 11:733348. doi: 10.3389/fcimb.2021.733348

\section{Application of Fatty Liver Inhibition of Progression Algorithm and Steatosis, Activity, and Fibrosis Score to Assess the Impact of Non-Alcoholic Fatty Liver on Untreated Chronic Hepatitis B Patients}

\author{
Yan Huang ${ }^{\dagger}$, Qinyi Gan ${ }^{\dagger}$, Rongtao Lai, Weijing Wang, Simin Guo, Zike Sheng, Lu Chen, \\ Qing Guo, Wei Cai, Hui Wang, Gangde Zhao*, Zhujun Cao* and Qing Xie* \\ Department of Infectious Diseases, Ruijin Hospital, Shanghai Jiao Tong University School of Medicine, Shanghai, China
}

Backgrounds and Purpose: Concurrent non-alcoholic fatty liver disease (NAFLD) in chronic hepatitis $\mathrm{B}(\mathrm{CHB})$ patients is a frequent and increasingly concerning problem because of the NAFLD pandemic. Admittedly, NAFLD can progress to non-alcoholic steatohepatitis (NASH) and severe fibrosis. Direct evidence of the fibrotic effect of NAFLD or NASH in chronic hepatitis B virus (HBV) infection remains lacking. We aimed to reveal the influence of concurrent histologically proven fatty liver diseases in fibrogenesis with chronic HBV infection.

Methods: We performed a retrospective cross-sectional study on a liver biopsy population of $\mathrm{CHB}$ patients without excessive alcohol intake to evaluate the prevalence of concurrent histologically proven NAFLD or NASH according to the fatty liver inhibition of progression (FLIP) algorithm and its association with the liver fibrosis stage.

Results: Among 1,081 CHB patients, concurrent NAFLD was found in 404 patients (37.4\%), among whom 24.0\% (97/404) have NASH. The presence of NASH was an independent predictor of significant fibrosis (odds ratio (OR), 2.53; 95\% Cl, 1.52-4.21; $p<$ $0.001)$ and severe fibrosis (OR, 1.83; 95\% Cl, 1.09-3.09; $p=0.023)$ in all patients, as well as in patients with normal alanine aminotransferase (ALT) (predicting significant fibrosis, $\mathrm{OR}, 2.86,95 \% \mathrm{Cl}, 1.34-6.10 ; p=0.007)$. The presence of lobular inflammation $(p<$ $0.001)$ or presence of cytological ballooning $(p<0.001)$, rather than presence of steatosis $(p=0.419)$, was related with severity of fibrosis in Spearman's correlation analysis.

Conclusions: Concurrent NAFLD is common in $\mathrm{CHB}$ patients, and $\mathrm{NASH}$ is an independent risk factor potentiating significant fibrosis by 2.53 -fold and severe fibrosis by 1.83-fold. While coping with chronic HBV infection, routine assessment of co-existing NAFLD or NASH is also important.

Keywords: hepatitis B, non-alcoholic fatty liver disease (NAFLD), non-alcoholic steatohepatitis (NASH), liver biopsy, liver fibrosis 


\section{INTRODUCTION}

As a major global public health problem, chronic hepatitis $\mathrm{B}$ (CHB) affects an estimated 292 million people (Ott et al., 2017; Polaris Observatory, 2018). With the increased incidence of obesity and metabolic syndrome, non-alcoholic fatty liver (NAFL) disease (NAFLD) has become another important cause of liver cirrhosis and hepatocellular carcinoma (HCC) worldwide, affecting roughly $25 \%$ of the world's population (Loomba and Sanyal, 2013; Younossi et al., 2016; Younossi et al., 2018). The disease spectrum of NAFLD ranges from NAFL to non-alcoholic steatohepatitis (NASH), which is histologically characterized by the presence of steatosis, inflammation, and ballooning. Because of their high prevalence and increased diagnosis of NAFLD, superimposed NAFLD in chronic hepatitis B virus (HBV) infection has been increasingly common in clinical practice nowadays.

It is reasonable to suppose that the development of NAFLD in $\mathrm{CHB}$ would exaggerate liver lesions and accelerate the remodeling of liver structure, leading to an increased risk of disease progression.

Previous studies have demonstrated that metabolic syndrome is an independent risk factor for fibrosis progression (Wong et al., 2009; Adams et al., 2017), cirrhosis, and poor clinical outcomes (Cheng et al., 2016) in CHB patients. A large multicenter cohort study revealed that patients with concomitant $\mathrm{NASH}$ and $\mathrm{CHB}$ have a higher risk of developing liver-related outcomes (Choi et al., 2020). However, several studies suggested that hepatic steatosis in CHB patients does not correlate with the activity of necroinflammation or stage of liver fibrosis (Thomopoulos et al., 2006; Bondini et al., 2007; Zheng et al., 2010). The impact of steatosis or steatohepatitis on disease progression remains inconclusive in these studies. Our hypothesis was that the presence of NASH in CHB patients was associated with an increased risk of significant and severe fibrosis.

The current study was aimed to test this hypothesis in a large cohort of $\mathrm{CHB}$ patients with liver biopsy for histological assessment.

\section{MATERIALS AND METHODS}

\section{Study Design}

This is a cross-sectional analysis of a prospectively collected dataset for patients receiving percutaneous liver biopsy in Shanghai Ruijin Hospital. Details of the biopsy procedure, data collection, and biobank maintenance were described previously (Cao et al., 2017a; Cao et al., 2017b). All the patients provided written informed consent for liver biopsy. The indications for liver biopsies are as follows: 1) staging of known parenchymal liver disease to predict prognosis; 2) instructing on whether to start antiviral therapy based on the histologic staging of inflammation and fibrosis, especially in patients with normal or mildly elevated alanine aminotransferase (ALT); and 3) diagnosis of other liver diseases other than HBV infection.
Currently, there is no established scoring system for assessing the activity of fatty liver in patients with CHB. For the purpose of analysis, a robust score, "steatosis, activity, and fibrosis (SAF) score" established (Bedossa and Consortium, 2014) and validated (Nascimbeni et al., 2020) in patients with NAFLD was used to evaluate steatosis, lobular inflammation, and ballooning degeneration. Based on the SAF score, patients were classified into three groups-NASH, NAFL, and no NAFLD-according to the fatty liver inhibition of progression (FLIP) algorithm. All the liver specimens from eligible patients were retrospectively assessed for the SAF score and FLIP classification. The current study complied with the Declaration of Helsinki and was approved by the ethics review committee of Shanghai Ruijin Hospital. Patient informed consent was waived by the committee considering the retrospective design.

\section{Patients}

All patients who received liver biopsy between January 2009 and December 2018 at our unit were assessed for eligibility. Inclusion criteria i) aged older than 16 years and ii) with positive serum hepatitis B surface antigen for at least 6 months. Exclusion criteria were i) with evidence of other chronic liver diseases including other viral hepatitis, autoimmune hepatitis, druginduced liver disease, and primary biliary cholangitis; ii) with evidence of HCC; iii) with previous history of antiviral therapy; iv) with unreliable scoring results derived from unqualified liver samples, defined as less than $10 \mathrm{~mm}$ or containing less than six portal triads; and v) with excessive alcohol assumption, defined as alcohol intake of $\geq 20 \mathrm{~g}$ per day for men and $\geq 10 \mathrm{~g}$ per day for women. A flowchart of the study design is illustrated in Supplementary Figure 1.

\section{Clinical Evaluation}

All patients received comprehensive clinical and laboratory assessments within 1 month before liver biopsy. Medical history of hypertension, diabetes mellitus (DM), alcohol consumption, and anthropometric parameters including body weight and body height were recorded. Overweight was defined as body mass index (BMI) $\geq 23 \mathrm{~kg} / \mathrm{m}^{2}$, and obesity as BMI $\geq 25$ $\mathrm{kg} / \mathrm{m}^{2}$, following Asian-specific recommendations (Consultation, 2004). The upper limit of normal (ULN) for ALT was defined as $\leq 40 \mathrm{U} / \mathrm{L}$ according to the European Association for the Study of the Liver (EASL) criteria (European Association for the Study of the Liver. Electronic address and European Association for the Study of the, 2017).

\section{Histological Evaluation}

Biopsy specimens were routinely stained with H\&E and Masson trichrome stains and assessed by two liver pathologists immediately after liver biopsies. Scheuer's scoring system was used for staging fibrosis (S0-S4) and inflammation (G0-G4) (Scheuer, 1991). Significant fibrosis was defined as $S \geq 2$ and severe fibrosis as $S \geq 3$. Steatosis, lobular inflammation, and cytological ballooning degeneration were reevaluated according to the SAF score by a liver pathologist blinded to clinical data/ biological data. According to the SAF scoring system, steatosis 
was scored on a $0-3$ scale, lobular inflammation on a $0-2$ scale, and ballooning on a 0-2 scale (Bedossa and Consortium, 2014). Histopathological lesions in NAFLD were assessed separately in the SAF score so that the SAF score was recommended and adopted to assess NAFLD in this article (Nascimbeni et al., 2020). Moderate-to-severe steatosis was defined as intrahepatic steatosis $\geq 33 \%$. Liver specimens with a length $\geq 10 \mathrm{~mm}$ or containing at least six portal triads were considered qualified for analysis, and the rest were excluded.

\section{Statistical Analysis}

Continuous variables were expressed in mean $\pm \mathrm{SD}$ or median (interquartile range [IQR]), as appropriate, whereas categorical variables were presented as number (percentage). Differences in continuous variables were examined for statistical significance using Student's t-test or Kruskal-Wallis rank-sum test depending on data distribution. Categorical variables were analyzed with the chi-squared test or Fisher's exact test. Correlation analyses were performed according to Spearman's method. Logistic regression analyses were used to identify risk factors for significant fibrosis or severe fibrosis. All the variables with a $p$-value less than 0.1 under univariate analysis entered the stepwise selection process, and those with a $p$-value less than 0.05 were retained. Variables were expressed as odds ratio (OR) and 95\% CI. $p$-Values $<0.05$ were considered statistically significant. We classified our patients into lean or non-lean groups according to the ethnic-specific BMI cutoff of $23 \mathrm{~kg} / \mathrm{m}^{2}$ in our current study with the Asian population. Subgroup analyses were performed by logistic regression. All data were analyzed using SAS (Version 9.4; SAS Institute Inc., Cary, NC, USA).

\section{RESULTS}

\section{Characteristics of the Study Population}

A total of 1,081 patients with treatment-naïve $\mathrm{CHB}$ were identified from 1,701 CHB patients who underwent a liver biopsy between 2009 and 2018 (Supplementary Figure 1), and patient characteristics are shown in Table 1. Among 1,081 treatment-naïve $\mathrm{CHB}$ patients, the median age was 37 years (IQR, 31-47), and $63.1 \%$ of them were males. Their median viral load was $5.33 \log 10 \mathrm{IU} / \mathrm{ml}$ (IQR, 3.74-7.18 $\log 10 \mathrm{IU} / \mathrm{ml}$ ), and $44.4 \%$ of them were $\mathrm{HBeAg-negative} \mathrm{patients.} \mathrm{The} \mathrm{overall}$ prevalence of diabetes, hypertension, and overweight was $1.5 \%$, $3.1 \%$, and $50.8 \%$, respectively. ALT elevation in this population was ubiquitous and accounted for $52.0 \%$ of all patients. The percentage of significant fibrosis $(S \geq 2)$ and severe fibrosis $(S \geq 3)$ according to Scheuer's scoring system was $50.1 \%$ and $22.9 \%$, respectively. The histopathological findings are illustrated in detail in Table $\mathbf{1 .}$

The FLIP algorithmic tree shows the number of patients in the different algorithmic pathways (Figure 1A). According to the FLIP algorithm, 62.6\% (677/1,081), 28.4\% (307/1,081), and 9.0\% $(97 / 1,081)$ CHB patients were classified as without NAFLD, with NAFL, and with NASH, respectively. Among all the patients with NAFLD, about a quarter $(24.0 \%, \mathrm{n}=97)$ of patients were diagnosed with NASH, among whom 17.5\% (17 of 97) patients were diagnosed with severe NASH (Figure 1B).

Compared with patients without NAFLD, patients with NAFL or NASH showed significantly higher median age, BMI, and ALT (all $p<0.01$ ) (Table 1). A higher proportion of male, diabetic, and arterial hypertensive patients were observed in patients with NAFLD than in those without (all $p<$ 0.01) (Table 1).

\section{Association Between Steatohepatitis and Severity of Liver Fibrosis}

The proportion of different fibrosis stages between NAFL and NASH subgroups was different (Table 1). Percentage of patients with significant fibrosis or severe fibrosis was significantly higher in NASH subgroups than those in NAFL subgroups $(74.2 \%$ vs. $38.8 \%, p<0.001$, or $35.1 \%$ vs. $24.8 \%, p=0.047$, respectively) (Table $\mathbf{1}$ ).

Factors associated with significant fibrosis or severe fibrosis are shown in Table 2. The presence of NASH, overweight, age, blood platelets count, ALT, hepatitis B surface antigen level, $\mathrm{HBeAg}$ status, and serum HBV DNA level were significantly associated with the presence of significant fibrosis (Table 2). Among these factors, the presence of NASH, overweight, age, and blood platelets count were independent predictors for significant fibrosis under a multivariable analysis. Using the same analytic strategy, we identified the presence of NASH, diabetes, overweight, blood platelets count, albumin, and HBV DNA level as independent factors associated with severe fibrosis (Table 2).

\section{Correlation Between Histological Changes and Severity of Liver Fibrosis}

Based on the significant association of significant/severe fibrosis with the presence of NASH, we further compared the distribution of fibrosis stages based on Scheuer's scoring system between patients with and without steatosis, lobular inflammation, or cytological ballooning and found that the proportion of significant fibrosis was significantly different between patient with or without lobular inflammation $(57.1 \%$ vs. $34.3 \%, p<0.001)$ and patients with or without cytological ballooning ( $75.1 \%$ vs. $44.6 \%, p<0.001)$ (Figure 2). The proportion of significant fibrosis showed no difference between patients with or without steatosis $(47.3 \%$ vs. $51.9 \%, p=0.146)$ (Figure 2). According to spearman correlation analyses, both the degree of lobular inflammation and degree of cytological ballooning were significantly associated with fibrosis stage (both $p<0.001$ ) (Table 3). The presence of steatosis, presence of moderate-to-severe steatosis, and degree of steatosis were not related to the fibrosis stage (all $p>0.05$ ).

\section{Factors Associated With Non-Alcoholic Steatohepatitis-Related Histological Changes}

Correlation analyses of metabolic and viral factors and NASHrelated histological changes were performed according to Spearman's method (Table 4). It was demonstrated that 
TABLE 1 | Demographic, clinical, and biochemical characteristics and liver pathological features of the patient population.

\begin{tabular}{|c|c|c|c|c|c|}
\hline Variable & All $(n=1,081)$ & No NAFLD $(n=677)$ & NAFL (n = 307) & NASH (n = 97) & $p$-Value ${ }^{1}$ \\
\hline Age (years) & $37(31-47)$ & $37(30-46)$ & $38(31-47)$ & $42(35-50)$ & $<0.001$ \\
\hline Male (n, \%) & $682(63.1 \%)$ & $387(57.2 \%)$ & $222(72.3 \%)$ & $62(77.5 \%)$ & $<0.001$ \\
\hline BMI $\left(\mathrm{kg} / \mathrm{m}^{2}\right)$ & $23.0(20.8-25.3)$ & $22.3(20.3-24.2)$ & $23.9(21.9-26.1)$ & $25.5(23.5-27.7)$ & $<0.001$ \\
\hline$<23$ (normal) (n, \%) & $512(49.2 \%)$ & $390(59.0 \%)$ & $104(36.8 \%)$ & $18(18.6 \%)$ & $<0.001$ \\
\hline 23-24.9 (overweight) (n, \%) & 430 (41.3\%) & 239 (36.2\%) & $139(49.1 \%)$ & $52(53.6 \%)$ & \\
\hline$\geq 25$ (obese) (n, \%) & $99(9.5 \%)$ & $32(4.8 \%)$ & $40(14.1 \%)$ & $27(27.8 \%)$ & \\
\hline Diabetes (n, \%) & $16(1.5 \%)$ & $5(0.7 \%)$ & $7(2.3 \%)$ & $4(4.1 \%)$ & 0.004 \\
\hline Arterial hypertension (n, \%) & $33(3.1 \%)$ & $14(2.1 \%)$ & $8(2.6 \%)$ & $11(11.3 \%)$ & $<0.001$ \\
\hline Family HCC history (n, \%) & $124(11.5 \%)$ & $69(10.2 \%)$ & $41(13.4 \%)$ & $83(85.6 \%)$ & 0.093 \\
\hline Platelets $\left(10^{9} / \mathrm{L}\right)$ & $172(142-206)$ & $172(143-202)$ & $168(140-208)$ & $186(147-216)$ & 0.242 \\
\hline Albumin (g/L) & $43(28-73)$ & $44(41-46)$ & $43(40-45)$ & $45(41-47)$ & 0.005 \\
\hline $\mathrm{TB}(\mu \mathrm{mol} / \mathrm{L})$ & $16(12-20)$ & $16(12-20)$ & $17(13-22)$ & $14(11-18)$ & 0.001 \\
\hline ALT (IU/L) & $42(28-73)$ & $40(26-71)$ & $45(31-78.5)$ & $43(28-62)$ & 0.008 \\
\hline ALT > $40 \mathrm{IU} / \mathrm{L}(\mathrm{n}, \%)$ & $528(52.0)$ & $314(48.9 \%)$ & $162(57.9 \%)$ & $52(55.3 \%)$ & 0.035 \\
\hline AST (IU/L) & $34(26-48)$ & $33(25-48)$ & $35(27-52)$ & $32.5(26-42)$ & 0.117 \\
\hline HBsAg (log IU/ml) & $3.51(3.00-4.17)$ & $3.51(2.98-4.16)$ & $3.52(3.01-4.34)$ & $3.43(3.13-4.01)$ & 0.890 \\
\hline HBeAg-positive (n, \%) & $582(55.6 \%)$ & $368(55.8 \%)$ & $177(61.0 \%)$ & $37(38.1 \%)$ & 0.084 \\
\hline HBV DNA (log IU/ml) & $5.34(3.72-7.18)$ & $5.40(3.74-7.25)$ & $5.57(3.92-7.15)$ & $4.38(3.31-5.98)$ & 0.002 \\
\hline \multicolumn{6}{|l|}{ HBV genotype, n (\%) } \\
\hline$B$ & $101(34.0 \%)$ & 68 (36.2\%) & 25 (29.1\%) & $8(34.8 \%)$ & \\
\hline C & 188 (63.3\%) & $119(63.3 \%)$ & 54 (62.8\%) & $15(65.2 \%)$ & 0.355 \\
\hline Other & $8(2.7 \%)$ & $1(0.5 \%)$ & $7(8.1 \%)$ & $0(0.0 \%)$ & \\
\hline Significant fibrosis (n, \%) & $542(50.1 \%)$ & 351 (51.9\%) & $119(38.8 \%)$ & $72(74.2 \%)$ & 0.273 \\
\hline Severe fibrosis (n, \%) & 247 (22.9\%) & $137(20.2 \%)$ & $76(24.8 \%)$ & $34(35.1 \%)$ & 0.001 \\
\hline \multicolumn{6}{|l|}{ Steatosis (n, \%) } \\
\hline $0,<5 \%$ & 677 (62.6\%) & 677 (100.0\%) & $0(0 \%)$ & $0(0 \%)$ & $<0.001$ \\
\hline $1,5 \%-33 \%$ & 308 (28.5\%) & $0(0 \%)$ & 239 (77.9\%) & $69(71.1 \%)$ & \\
\hline $2,33 \%-66 \%$ & $79(7.3 \%)$ & $0(0 \%)$ & $54(17.6 \%)$ & 25 (25.8\%) & \\
\hline $3, \geq 66 \%$ & $17(1.6 \%)$ & $0(0 \%)$ & $14(4.6 \%)$ & $3(3.1 \%)$ & \\
\hline \multicolumn{6}{|l|}{ Lobular inflammation (n, \%) } \\
\hline 0, no foci & 332 (30.7\%) & $193(28.5 \%)$ & $139(45.3 \%)$ & $0(0 \%)$ & 0.172 \\
\hline $1,<2$ foci & 669 (61.9\%) & $435(64.3 \%)$ & $145(47.2 \%)$ & $89(91.2 \%)$ & \\
\hline $2, \geq 2$ foci & $80(7.4 \%)$ & $49(7.2 \%)$ & $23(7.5 \%)$ & 8 (8.3\%) & \\
\hline \multicolumn{6}{|l|}{ Cytological ballooning (n, \%) } \\
\hline 0, none & $884(81.8 \%)$ & $582(86.0 \%)$ & 302 (98.4\%) & $0(0 \%)$ & $<0.001$ \\
\hline 1, few & $186(17.2 \%)$ & $93(13.7 \%)$ & $5(1.6 \%)$ & $88(90.7 \%)$ & \\
\hline 2, many & $11(1.02 \%)$ & $2(0.3 \%)$ & $0(0 \%)$ & $9(9.3 \%)$ & \\
\hline
\end{tabular}

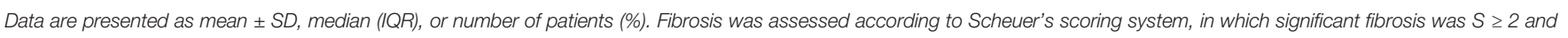
severe fibrosis was $S \geq 3$.

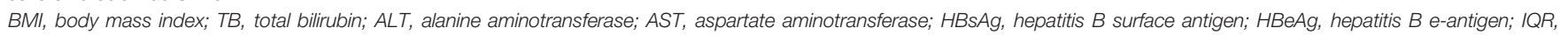

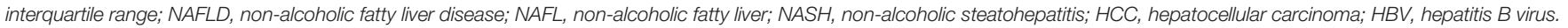
${ }^{1} \mathrm{p}$-Value for comparison between 3 groups of no NAFLD, NAFL, and NASH.

metabolic factors (including BMI, diabetes, and hypertension) were mildly correlated with the degree of steatosis or cytological ballooning but not lobular inflammation. Among viral factors, HBV DNA but not HBsAg or HBeAg status was associated with lobular inflammation. Clinical factors related to lobular inflammation only included ALT and AST.

\section{Subgroup Analysis of the Association Between Non-Alcoholic Steatohepatitis and Significant Fibrosis}

To further analyze the association between NASH and significant fibrosis in different populations, we performed univariable analyses and multivariable analyses in subgroups (HBeAgpositive, HBeAg-negative, ALT $\leq \mathrm{ULN}, \mathrm{ALT}>\mathrm{ULN}$, and BMI $<23 \mathrm{~kg} / \mathrm{m}^{2}$ ). The results showed that NASH was independently related to significant fibrosis in four subgroups ( $p=0.004$ for HBeAg-positive; $p=0.008$ for HBeAg-negative; $p=$
0.007 for ALT $\leq \mathrm{ULN} ; p=0.038$ for ALT $>$ ULN) (Tables 5 and 6).

Moreover, 512 (47.4\%) patients were classified as lean $\mathrm{CHB}$, among whom $122(23.8 \%)$ and 18 (3.5\%) patients had concomitant NAFLD or NASH, respectively according to the FLIP algorithm (Supplementary Figure 2). Patient characteristics and pathological features according to the presence of NAFLD or NASH are enumerated in Supplementary Table 3. Similar to the results in the entire cohort, significant fibrosis was more frequently presented in lean patients with lobular inflammation $(52.7 \%$ vs. $27.9 \%, p<0.01)$, or with cytological ballooning ( $68.9 \%$ vs. $41.5 \%, p<0.01)$ but not with steatosis $(47.3 \%$ vs. $53.6 \%, p=0.171)$ than those without (Supplementary Figure 3). The OR of significant fibrosis and severe fibrosis in this specific population was 2.55 (95\% CI $0.94-$ 6.91, $p=0.065)$ and $3.06(95 \%$ CI $1.15-8.13, p=$ $0.025)$, respectively. 


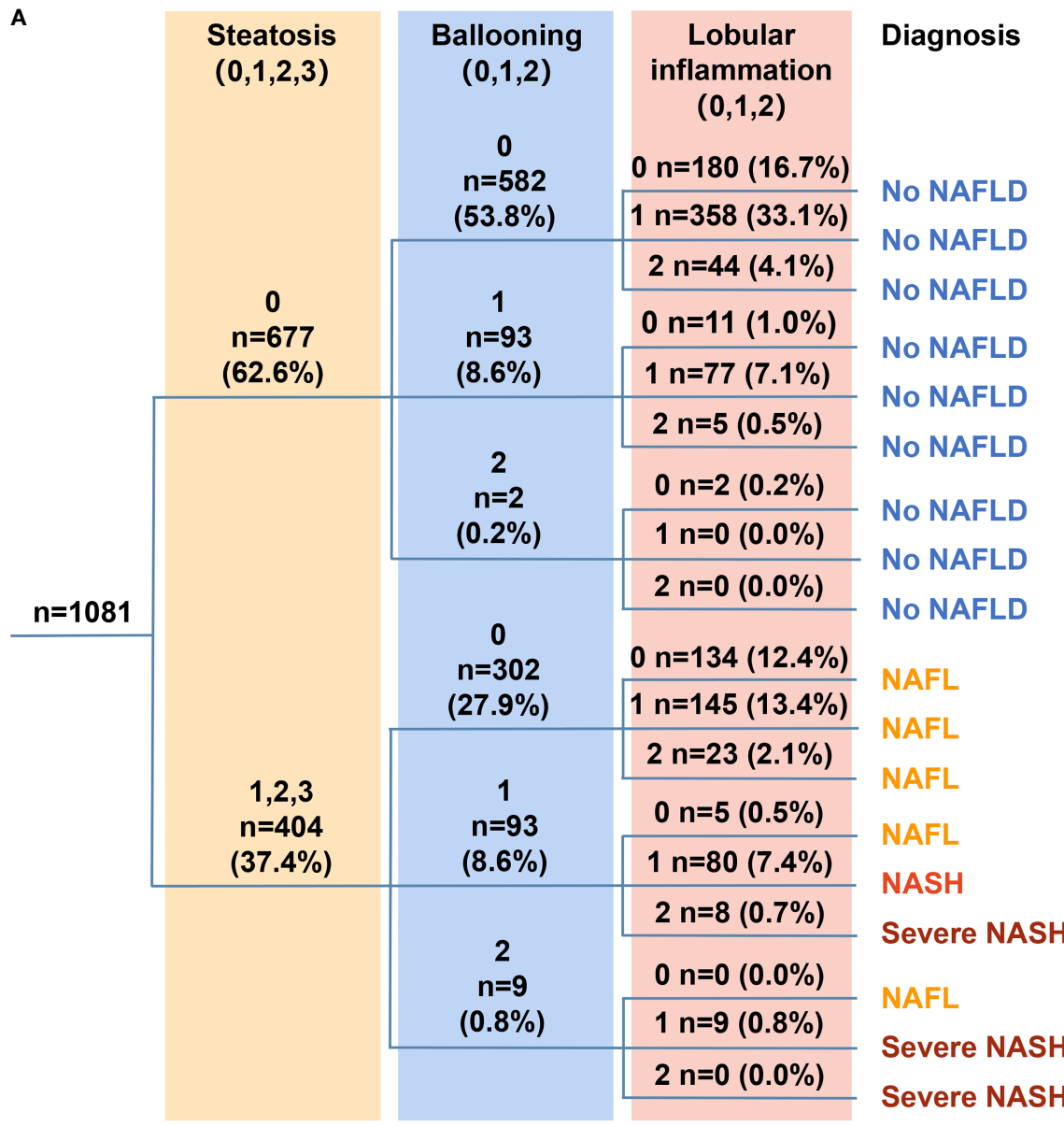

B

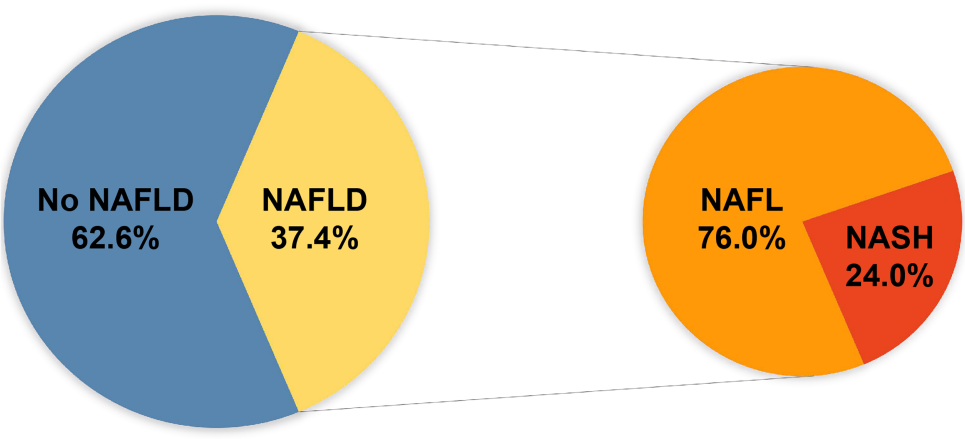

FIGURE 1 (A) The FLIP algorithmic tree. A total of 1,081 patients with treatment-naïve chronic hepatitis B were classified into no NAFLD, NAFL, NASH, or severe NASH according to the FLIP algorithmic pathways based on the grade of steatosis [(A); light yellow shade], grade of ballooning [(B); light blue shade], and grade of lobular inflammation [(A); pink shade]. Number of patients and the proportion among each category were presented as number (\%). (B) Pie chart illustrates the proportion of patients with specific diagnoses. Among all 1,081 patients, $62.6 \%$ had no NAFLD, and the remaining 37.4\% had NAFLD, among whom 76.0\% had NAFL and 24.0\% had NASH. FLIP, fatty liver inhibition of progression; NAFLD, non-alcoholic fatty liver disease; NAFL, non-alcoholic fatty liver; NASH, non-alcoholic steatosis hepatitis.

\section{DISCUSSION}

With the increasing prevalence of NAFLD, more and more attention is focused on the effects of NAFLD or NASH on disease progression in $\mathrm{CHB}$ infection. This study retrospectively investigated the biopsy-proven NAFLD/NASH as per the FLIP algorithm (Nascimbeni et al., 2020) in consecutive antiviral-naïve CHB patients with the largest sample size up to date (Thomopoulos et al., 2006; Bondini et al., 2007; Zheng et al., 2010; Charatcharoenwitthaya et al., 2017; 
TABLE 2 | Factors associated with significant fibrosis and severe fibrosis among patients with chronic HBV infection.

\begin{tabular}{|c|c|c|c|c|c|c|c|c|}
\hline \multirow[t]{3}{*}{ Variable } & \multicolumn{4}{|c|}{ Significant fibrosis $(\mathrm{S} \geq 2)$} & \multicolumn{4}{|c|}{ Severe fibrosis $(\mathbf{S} \geq 3$ ) } \\
\hline & \multicolumn{2}{|c|}{ Univariable analysis } & \multicolumn{2}{|c|}{ Multivariable analysis } & \multicolumn{2}{|c|}{ Univariable analysis } & \multicolumn{2}{|c|}{ Multivariable analysis } \\
\hline & OR (95\% Cl) & $p$-Value & OR $(95 \% \mathrm{Cl})$ & $p$-Value & OR (95\% Cl) & $p$-Value & OR $(95 \% \mathrm{Cl})$ & $p$-Value \\
\hline Age (years) & $1.03(1.01-1.04)$ & $<0.001$ & $1.02(1.01-1.03)$ & 0.004 & $1.04(1.02-1.05)$ & $<0.001$ & & \\
\hline Male (yes/no) & $1.17(0.92-1.50)$ & 0.205 & & & $1.18(0.87-1.59)$ & 0.282 & & \\
\hline Diabetes (yes/no) & $1.67(0.60-4.63)$ & 0.324 & & & $4.47(1.65-12.12)$ & 0.003 & $4.10(1.14-14.74)$ & 0.031 \\
\hline Hypertension (yes/no) & $1.55(0.76-3.15)$ & 0.225 & & & $1.08(0.48-2.43)$ & 0.847 & & \\
\hline Overweight (yes/no) & $1.66(1.30-2.12)$ & $<0.001$ & $1.50(1.13-1.99)$ & 0.005 & $1.82(1.36-2.44)$ & $<0.001$ & $1.80(1.27-2.54)$ & $<0.001$ \\
\hline Family HCC history (yes/no) & $1.08(0.74-1.57)$ & 0.679 & & & $1.07(0.68-1.69)$ & 0.782 & & \\
\hline Platelets $\left(10^{9} / \mathrm{L}\right)$ & $0.99(0.99-0.99)$ & $<0.001$ & $0.99(0.99-1.00)$ & $<0.001$ & $0.98(0.98-0.99)$ & $<0.001$ & $0.98(0.98-0.99)$ & $<0.001$ \\
\hline Albumin (g/L) & $0.99(0.96-1.03)$ & 0.687 & & & $0.92(0.88-0.95)$ & $<0.001$ & $0.93(0.89-0.97)$ & 0.002 \\
\hline TB $(\mu \mathrm{mol} / \mathrm{L})$ & $1.04(0.99-1.02)$ & 0.387 & & & $1.00(0.99-1.01)$ & 0.854 & & \\
\hline $\operatorname{ALT}(\mathrm{IU} / \mathrm{L})$ & $1.00(1.00-1.00)$ & 0.046 & & & $1.00(1.00-1.00)$ & 0.242 & & \\
\hline AST (IU/L) & $1.00(1.00-1.00)$ & 0.823 & & & $1.00(1.00-1.00)$ & 0.539 & & \\
\hline HBsAg (log IU/ml) & $0.80(0.68-0.95)$ & 0.009 & & & $0.78(0.65-0.93)$ & 0.006 & & \\
\hline HBeAg-positive (yes/no) & $0.76(0.59-0.97)$ & 0.025 & & & $0.76(0.57-1.02)$ & 0.068 & & \\
\hline HBV DNA (log IU/ml) & $0.85(0.82-0.93)$ & $<0.001$ & & & $0.86(0.80-0.93)$ & $<0.001$ & $0.88(0.82-0.95)$ & $<0.001$ \\
\hline Presence of NAFLD (yes/no) & $0.83(0.65-1.07)$ & 0.147 & & & $1.48(1.11-1.97)$ & 0.008 & & \\
\hline Presence of NASH (yes/no) & $3.02(1.90-4.82)$ & $<0.001$ & $2.53(1.52-4.21)$ & $<0.001$ & $1.95(1.25-3.05)$ & 0.003 & 1.83 (1.09-3.09) & 0.023 \\
\hline
\end{tabular}

Fibrosis was assessed according to Scheuer's scoring system, in which significant fibrosis was $S \geq 2$ and severe fibrosis was $S \geq 3$.

Overweight, body mass index $\geq 23 \mathrm{~kg} / \mathrm{m}^{2}$; TB, total bilirubin; ALT, alanine aminotransferase; AST, aspartate aminotransferase; HBV, hepatitis B virus; HCC, hepatocellular carcinoma; NAFLD, non-alcoholic fatty liver disease; NAFL, non-alcoholic fatty liver; NASH, non-alcoholic steatohepatitis.

Shen et al., 2019). We identified $37.4 \%$ of patients with co-existing NAFLD (intrahepatic steatosis $\geq 5 \%$ ) and $24.0 \%$ of patients who have concomitant NAFLD were diagnosed with NASH. The incidence of NAFLD in CHB patients is controversial. It has been reported that $\mathrm{CHB}$ patients had a lower incidence of NAFLD (Zhong et al., 2018). In Zhong's study, NAFLD was diagnosed by abdominal ultrasonography, which is not sensitive to mild steatosis. Other studies reported that the prevalence of NAFLD detected by liver biopsy was $37.6 \%$ (Shen et al., 2019) or 38.3\% (Charatcharoenwitthaya et al., 2017), which is similar to our results. What is more, lobular inflammation and cytological ballooning had significant effects on the severity of liver fibrosis, but the presence of steatosis did not, which reinforced the conclusion that inflammation activity and hepatocyte lesion caused by excessive intrahepatic steatosis deposition are chief culprits responsible for fibrosis progression in CHB patients (Machado and Diehl, 2016). Among patients with normal ALT, the occurrence of NASH is strongly associated with significant fibrosis. Such effect was independent of HBeAg status. Exactly as a previous study shows that the proportion of NASH patients with normal ALT in overall NASH patients was 19\% (95\% CI 13\%-27\%) (Ma et al., 2020), ALT is not sensitive in
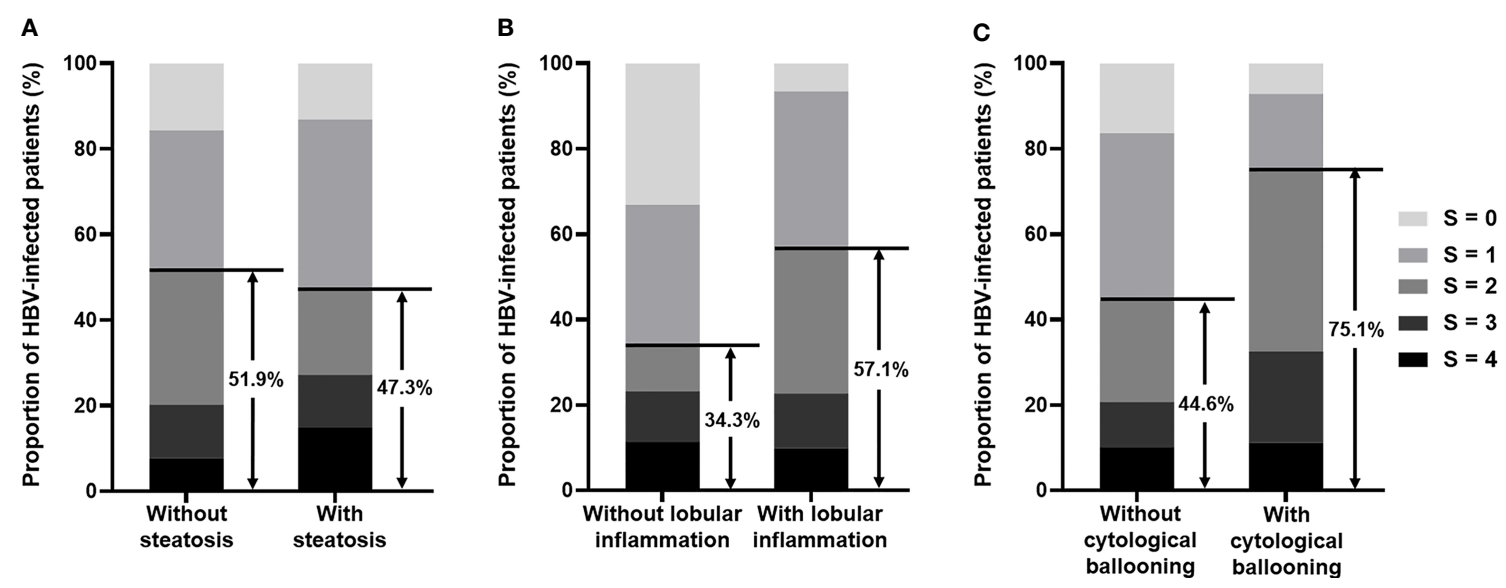

FIGURE 2 | (A) Bar chart illustrates the proportion of fibrosis stage in HBV-infected patients with or without steatosis. Among patients without steatosis, 51.9\% had significant fibrosis $(S \geq 2)$, and among patients with steatosis, $47.3 \%$ had significant fibrosis $(S \geq 2)$. (B) Bar chart illustrates the proportion of fibrosis stage in HBVinfected patients with or without lobular inflammation. Among patients without lobular inflammation, $34.3 \%$ had significant fibrosis (S $\geq 2)$, and among patients with lobular inflammation, $57.1 \%$ had significant fibrosis ( $\mathrm{S} \geq 2$ ). (C) Bar chart illustrates the proportion of fibrosis stage in HBV-infected patients with or without cytological ballooning. Among patients without cytological ballooning, $44.6 \%$ had significant fibrosis $(S \geq 2)$, and among patients with cytological ballooning, $75.1 \%$ had significant fibrosis $(\mathrm{S} \geq 2)$. HBV, hepatitis B virus. 
TABLE 3 | Spearman's correlation analysis between three histological changes and severity of fibrosis.

Variable
Fibrosis stage

\begin{tabular}{cc}
\hline rho & $\boldsymbol{p}$-Value \\
0.025 & 0.419 \\
0.024 & 0.436 \\
0.026 & 0.386 \\
0.208 & $<0.001$ \\
0.209 & $<0.001$ \\
0.199 & $<0.001$ \\
0.194 & $<0.001$
\end{tabular}

Fibrosis was assessed according to Scheuer's scoring system.

predicting the presence of NASH. The hidden fibrotic influence of NASH among patients with normal liver function has always been neglected.

Meanwhile, we found $484(44.8 \%)$ patients with no steatosis but with lobular inflammation in this study. It could be related to HBV itself. These HBV patients might be categorized as having HBV-related lobular activity. Histological lesions such as spotty lobular inflammation are common in $\mathrm{CHB}$ and, therefore, are not specific for steatohepatitis (Mani and Kleiner, 2009). It is exceedingly difficult to affirm the overlap of viral hepatitis and steatohepatitis in histological activity. Peng et al. found steatosis to be more frequent in CHB than in the general population, and they hypothesize that this may be due to metabolic factors or the ability of HBV to indirectly facilitate the development of steatosis (Peng et al., 2008). On the other hand, some studies demonstrated that HBV infection may exacerbate metabolic dysregulation in NAFLD in animal and cell models. Kim et al. found that HBV participates in the activation of SREBP1 and peroxisome proliferator-activated receptor- $\gamma$ (PPAR- $\gamma$ ) (Kim et al., 2007; Kim et al., 2008). HBV infection may exacerbate metabolic dysregulation in NAFLD as suggested by previous studies based on animal models (Zhang et al., 2020). The interaction between $\mathrm{HBV}$ infection and steatosis still needs to be fully elucidated in the future. On the other hand, there are different opinions about concurrent NAFLD downregulating the viral titer in $\mathrm{CHB}$ patients. A previous study in Hong Kong reported the inverse relationship between hepatic steatosis $(\mathrm{CAP} \geq 222 \mathrm{~dB} / \mathrm{m})$ and hepatitis $\mathrm{B}$ viremia in treatment-naïve $\mathrm{CHB}$ patients by multivariable logistic analysis $(p=0.041$; OR, 0.859, 95\% CI 0.743-0.994) (Hui et al., 2018). However, Charatcharoenwitthaya et al. thought that there is no association between HBV DNA and histological-proven steatosis $(p=0.599)$ in the Asian population according to a univariable logistic analysis (Charatcharoenwitthaya et al., 2017). Our data verified the result and demonstrated that the presence of steatosis was correlated with metabolic factors (including BMI, diabetes, and hypertension), rather than HBV DNA level $(p=0.310)$. The bidirectional correlation of CHB and NAFLD is complicated and is worthy of future study.

Our findings are of important clinical implications. First, we reproved that liver fibrosis in $\mathrm{CHB}$ patients concurrent with $\mathrm{NASH}$ is severer than in CHB patients without NASH. Attention should be focused on routine assessment for concomitance of NAFLD/NASH apart from management of HBV infection. Second, a wider indication for liver biopsies should be considered among patients who are highly suspected to have concomitant steatohepatitis. At present, no available noninvasive test (NIT) has acceptable accuracy, and liver biopsy remains the reference standard for the diagnosis of NASH (European Association for the Study of the Liver. Electronic address et al., 2021). Since the presence of NASH was associated with fibrosis progression among patients with normal ALT, more concerns are needed for patients with normal ALT who were universally thought to have no or mild histological changes in the liver. As the latest EASL Clinical Practice Guidelines pointed out, automatic calculation and systematic reporting of simple noninvasive fibrosis tests such as Fibrosis-4 (FIB-4) and routine detection of liver stiffness by transient elastography are beneficial to screening for liver fibrosis currently (European Association for the Study of the Liver. Electronic address et al., 2021). Lastly, early recognition and management of NAFLD and its associated metabolic disorders in $\mathrm{CHB}$ patients may bring a beneficial effect

TABLE 4 | Correlation analysis between metabolic, virological factors, and NASH-related histological changes.

\begin{tabular}{|c|c|c|c|c|c|c|}
\hline \multirow[t]{2}{*}{ Variable } & \multicolumn{2}{|c|}{ Degree of steatosis } & \multicolumn{2}{|c|}{ Degree of lobular inflammation } & \multicolumn{2}{|c|}{ Degree of cytological ballooning } \\
\hline & rho & $p$-Value & rho & $p$-Value & rho & $p$-Value \\
\hline Diabetes (yes/no) & 0.079 & 0.009 & 0.022 & 0.475 & 0.064 & 0.037 \\
\hline Hypertension (yes/no) & 0.078 & 0.011 & 0.041 & 0.180 & 0.137 & $<0.001$ \\
\hline $\mathrm{BMI}\left(\mathrm{kg} / \mathrm{m}^{2}\right)$ & 0.315 & $<0.001$ & 0.052 & 0.095 & 0.189 & $<0.001$ \\
\hline HBsAg (log IU/ml) & 0.015 & 0.716 & 0.046 & 0.249 & -0.043 & 0.286 \\
\hline HBeAg-positive (yes/no) & -0.011 & 0.720 & 0.060 & 0.052 & -0.129 & $<0.001$ \\
\hline HBV DNA (log IU/ml) & -0.042 & 0.182 & 0.124 & $<0.001$ & -0.097 & 0.002 \\
\hline
\end{tabular}

BMI, body mass index; HBsAg, hepatitis B surface antigen; NASH, non-alcoholic steatohepatitis; HBeAg, hepatitis B e-antigen; HBV, hepatitis B virus. 
TABLE 5 | Univariable analyses and multivariable analyses of factors associated with significant fibrosis in HBeAg-positive and HBeAg-positive subgroups.

\begin{tabular}{|c|c|c|c|c|c|c|c|c|}
\hline \multirow[t]{3}{*}{ Variable } & \multicolumn{4}{|c|}{ HBeAg-positive ( $n=582$ ) } & \multicolumn{4}{|c|}{ HBeAg-positive ( $n=464$ ) } \\
\hline & \multicolumn{2}{|c|}{ Univariable analysis } & \multicolumn{2}{|c|}{ Multivariable analysis } & \multicolumn{2}{|c|}{ Univariable analysis } & \multicolumn{2}{|c|}{ Multivariable analysis } \\
\hline & OR (95\% CI) & $p$-Value & OR (95\% Cl) & $p$-Value & OR $(95 \% \mathrm{Cl})$ & $p$-Value & OR $(95 \% \mathrm{Cl})$ & $p$-Value \\
\hline Age (years) & $1.03(1.03-1.05)$ & $<0.001$ & & & 1.01 (0.99-1.03) & 0.200 & & \\
\hline Male (yes/no) & $1.12(0.80-1.57)$ & 0.506 & & & $1.25(0.85-1.84)$ & 0.253 & & \\
\hline Diabetes (yes/no) & $1.36(0.36-5.13)$ & 0.647 & & & $4.12(0.48-35.57)$ & 0.198 & & \\
\hline Hypertension (yes/no) & $3.31(0.66-16.53)$ & 0.145 & & & $0.96(0.42-2.19)$ & 0.919 & & \\
\hline Overweight (yes/no) & $1.86(1.33-2.61)$ & $<0.001$ & $1.76(1.20-2.58)$ & 0.004 & $1.59(1.09-2.31)$ & 0.016 & & \\
\hline Family HCC history (yes/no) & $1.17(0.69-1.98)$ & 0.557 & & & $1.22(0.71-2.11)$ & 0.475 & & \\
\hline Platelets $\left(10^{9} / \mathrm{L}\right)$ & $0.99(0.99-0.99)$ & $<0.001$ & 0.99 (0.99-0.99) & $<0.001$ & $0.99(0.99-1.00)$ & $<0.001$ & $0.99(0.99-1.00)$ & $<0.001$ \\
\hline Albumin (g/L) & $0.96(0.92-1.00)$ & 0.077 & & & $1.02(0.97-1.08)$ & 0.357 & & \\
\hline TB $(\mu \mathrm{mol} / \mathrm{L})$ & $1.01(0.99-1.02)$ & 0.404 & & & 1.00 (0.98-1.03) & 0.957 & & \\
\hline ALT (IU/L) & $1.00(1.00-1.00)$ & 0.058 & & & $1.00(1.00-1.01)$ & 0.522 & & \\
\hline AST (IU/L) & $1.00(1.00-1.00)$ & 0.428 & & & 1.01 (1.00-1.02) & 0.037 & & \\
\hline HBsAg (log IU/ml) & 0.59 (0.45-0.78) & $<0.001$ & & & 1.25 (0.96-1.62) & 0.099 & & \\
\hline HBV DNA (log IU/ml) & $0.73(0.65-0.82)$ & $<0.001$ & $0.78(0.68-0.89)$ & $<0.001$ & $1.12(0.97-1.29)$ & 0.130 & & \\
\hline Presence of NAFLD & $1.07(0.77-1.51)$ & 0.678 & & & $0.61(0.42-0.89)$ & 0.010 & & \\
\hline Presence of NASH & $3.64(1.69-7.87)$ & 0.001 & 3.38 (1.47-7.79) & 0.004 & $2.49(1.36-4.56)$ & 0.003 & $2.32(1.24-4.34)$ & 0.008 \\
\hline
\end{tabular}

Fibrosis was assessed according to Scheuer's scoring system, in which significant fibrosis was $S \geq 2$.

Overweight, BMI $\geq 23 \mathrm{~kg} / \mathrm{m}^{2}$; TB, total bilirubin; ALT, alanine aminotransferase; AST, aspartate aminotransferase; HBsAg, hepatitis B surface antigen; HBeAg, hepatitis B e-antigen; OR, odds ratio; HCC, hepatocellular carcinoma; HBV, hepatitis B virus.

on their long-term outcomes. To CHB patients with NASH, the treatment strategy should include treatment of metabolic dysfunction and drug therapies targeting NASH when available, besides weight loss and lifestyle modifications.

Our study is limited by the retrospective design. The lack of data, including dyslipidemia, waist-hip ratios, and blood pressure levels, precludes us from making a more convincing conclusion. Moreover, the evaluation of the SAF score was not cross-validated by two or more pathologists, and complete pathological assessment including assessment of portal inflammation and lack of confluent necrosis might strengthen and enrich our conclusions. A well- developed prospective cohort study to further investigate the influence of NASH in fibrosis progression and long-term outcomes among $\mathrm{CHB}$ patients will be needed.

In conclusion, NAFLD is common in chronic HBV-infected patients, while NASH is associated with an increased risk of significant or severe fibrosis, suggesting a contributive effect on the pathogenesis of disease progression in $\mathrm{CHB}$ patients. More concern should be paid to the assessment of steatohepatitis and overweight besides managing CHB. Liver biopsies are recommended for $\mathrm{CHB}$ patients who are highly suspected of having co-existing NASH. Efforts on ameliorating steatohepatitis

TABLE 6 | Univariable analyses and multivariable analyses of factors associated with significant fibrosis among patients with normal ALT or abnormal ALT.

\begin{tabular}{|c|c|c|c|c|c|c|c|c|}
\hline \multirow[t]{3}{*}{ Variable } & \multicolumn{4}{|c|}{ ALT $\leq$ ULN $(n=347)$} & \multicolumn{4}{|c|}{ ALT > ULN $(n=669)$} \\
\hline & \multicolumn{2}{|c|}{ Univariable analysis } & \multicolumn{2}{|c|}{ Multivariable analysis } & \multicolumn{2}{|c|}{ Univariable analysis } & \multicolumn{2}{|c|}{ Multivariable analysis } \\
\hline & OR $(95 \% \mathrm{Cl})$ & $p$-Value & OR $(95 \% \mathrm{Cl})$ & $p$-Value & OR (95\% CI) & $p$-Value & OR $(95 \% \mathrm{Cl})$ & $p$-Value \\
\hline Age (years) & $1.02(1.00-1.04)$ & 0.019 & & & $1.04(1.02-1.06)$ & $<0.001$ & $1.02(1.00-1.04)$ & 0.042 \\
\hline Male (yes/no) & $1.13(0.79-1.61)$ & 0.614 & & & $1.18(0.81-1.72)$ & 0.403 & & \\
\hline Diabetes (yes/no) & $5.17(0.60-44.56)$ & 0.135 & & & $0.93(0.23-3.74)$ & 0.914 & & \\
\hline Hypertension (yes/no) & $1.14(0.45-2.84)$ & 0.787 & & & $2.12(0.65-6.98)$ & 0.215 & & \\
\hline Overweight (yes/no) & $1.90(1.31-2.75)$ & $<0.001$ & $1.76(1.20-2.58)$ & 0.007 & $1.59(1.09-2.31)$ & 0.007 & & \\
\hline Family HCC history (yes/no) & $1.17(0.66-2.08)$ & 0.601 & & & 1.09 (0.66-1.82) & 0.738 & & \\
\hline Platelets $\left(10^{9} / \mathrm{L}\right)$ & $0.99(0.99-1.00)$ & $<0.001$ & 0.99 (0.99-0.99) & $<0.001$ & 0.99 (0.98-0.99) & $<0.001$ & $0.99(0.98-0.99)$ & $<0.001$ \\
\hline Albumin (g/L) & $1.02(0.98-1.07)$ & 0.359 & & & $0.97(0.92-1.01)$ & 0.140 & & \\
\hline $\mathrm{TB}(\mu \mathrm{mol} / \mathrm{L})$ & $1.00(0.98-1.02)$ & 0.739 & & & $1.01(0.99-1.02)$ & 0.452 & & \\
\hline ALT (IU/L) & $1.02(1.00-1.05)$ & 0.081 & & & $1.00(1.00-1.00)$ & 0.006 & & \\
\hline AST (IU/L) & $1.05(1.02-1.07)$ & $<0.001$ & $1.04(1.01-1.07)$ & 0.006 & $1.00(1.00-1.00)$ & 0.327 & & \\
\hline HBsAg (log IU/ml) & $0.95(0.76-1.18)$ & 0.629 & & & $0.65(0.50-0.84)$ & $<0.001$ & & \\
\hline HBV DNA (log IU/ml) & $0.91(0.83-1.00)$ & 0.060 & & & $0.78(0.71-0.87)$ & $<0.001$ & $0.83(0.74-0.93)$ & 0.001 \\
\hline Presence of NAFLD & $0.82(0.56-1.18)$ & 0.304 & & & $0.80(0.57-1.13)$ & 0.211 & & \\
\hline Presence of NASH & $3.14(1.54-6.40)$ & 0.002 & $2.86(1.34-6.10)$ & 0.007 & $2.76(1.46-5.23)$ & 0.002 & $2.09(1.04-4.18)$ & 0.038 \\
\hline
\end{tabular}

Fibrosis was assessed according to Scheuer's scoring system, in which significant fibrosis was $S \geq 2$.

Overweight, BMI $\geq 23 \mathrm{~kg} / \mathrm{m}^{2}$; TB, total bilirubin; ALT, alanine aminotransferase; AST, aspartate aminotransferase; ULN, upper limit of normal; HBsAg, hepatitis B surface antigen; HCC, hepatocellular carcinoma; NAFLD, non-alcoholic fatty liver disease; NASH, non-alcoholic steatohepatitis; HBV, hepatitis B virus. 
by current approaches available are urgent in $\mathrm{CHB}$ patients who have concomitant NASH.

\section{DATA AVAILABILITY STATEMENT}

The raw data supporting the conclusions of this article will be made available by the authors, without undue reservation.

\section{ETHICS STATEMENT}

The studies involving human participants were reviewed and approved by Ruijin Hospital Ethics Committee, Shanghai Jiao Tong University School of Medicine. Written informed consent for participation was not required for this study in accordance with the national legislation and the institutional requirements.

\section{AUTHOR CONTRIBUTIONS}

QX, ZC, and YH conceived and designed the study. YH, ZC, QYG, RL, WW, SG, ZS, LC, and GZ collected the data. YH conducted the analysis and drafted the manuscript. QX, ZC, GZ, HW, and WC interpreted the data. QX, ZC, and QYG critically revised the manuscript. All authors contributed to the article and approved the submitted version.

\section{FUNDING}

This work was supported by the National Natural Science Foundation of China (No. 81570535 and No. 81770587), the Shanghai Three-Year Plan of the Clinical Skills and Innovations (16CR1002A), the Shanghai Municipal Key Clinical Specialty

\section{REFERENCES}

Adams, L. A., Anstee, Q. M., Tilg, H., and Targher, G. (2017). Non-Alcoholic Fatty Liver Disease and Its Relationship With Cardiovascular Disease and Other Extrahepatic Diseases. Gut 66, 1138-1153. doi: 10.1136/gutjnl-2017-313884

Bedossa, P.Consortium, F.P. (2014). Utility and Appropriateness of the Fatty Liver Inhibition of Progression (FLIP) Algorithm and Steatosis, Activity, and Fibrosis (SAF) Score in the Evaluation of Biopsies of Nonalcoholic Fatty Liver Disease. Hepatology 60, 565-575. doi: 10.1002/hep.27173

Bondini, S., Kallman, J., Wheeler, A., Prakash, S., Gramlich, T., Jondle, D. M., et al. (2007). Impact of Non-Alcoholic Fatty Liver Disease on Chronic Hepatitis B. Liver Int. 27, 607-611. doi: 10.1111/j.1478-3231.2007.01482.x

Cao, Z., Li, Z., Wang, Y., Liu, Y., Mo, R., Ren, P., et al. (2017b). Assessment of Serum Golgi Protein 73 as a Biomarker for the Diagnosis of Significant Fibrosis in Patients With Chronic HBV Infection. J. Viral Hepat. 24 (Suppl 1), 57-65. doi: $10.1111 /$ jvh.12786

Cao, Z., Li, Z., Wang, H., Liu, Y., Xu, Y., Mo, R., et al. (2017a). Algorithm of Golgi Protein 73 and Liver Stiffness Accurately Diagnoses Significant Fibrosis in Chronic HBV Infection. Liver Int. 37, 1612-1621. doi: 10.1111/liv.13536

Charatcharoenwitthaya, P., Pongpaibul, A., Kaosombatwattana, U., Bhanthumkomol, P., Bandidniyamanon, W., Pausawasdi, N., et al. (2017). The Prevalence of Steatohepatitis in Chronic Hepatitis B Patients and Its (shslczdzk01103), and the Shanghai Three-Year Plan of the Key Subjects Construction in Public Health-Infectious Diseases and Pathogenic Microorganism (15GWZK0102).

\section{SUPPLEMENTARY MATERIAL}

The Supplementary Material for this article can be found online at: https://www.frontiersin.org/articles/10.3389/fcimb.2021. 733348/full\#supplementary-material

Supplementary Figure 1 | Patients recruitment. Patients with NASH was diagnosed as SAF Score; HCC: hepatocellular carcinoma; excessive alcohol consumption: defined as alcohol intake $\geq 20 \mathrm{~g}$ per day for men and $\geq 10 \mathrm{~g}$ per day for women; poor quality liver samples: defined as liver sample less than $10 \mathrm{~mm}$ in length or containing less than six portal triads.

Supplementary Figure 2 | (A) FLIP algorithmic tree in lean population. A total of 512 patients with treatment naïve chronic hepatitis B with $\mathrm{BMl} \leq 23 \mathrm{~kg} / \mathrm{m}^{2}$ were classified into no NAFLD, NAFL, NASH or severe NASH according to the FLIP algorithmic pathways based on the grade of steatosis (Panel a, light yellow shade), grade of Ballooning (Panel a, light blue shade) and grade of lobular inflammation (Panel a, pink shade). Number of patients and the proportion among each category were presented as number (\%). (B) Pie chart to illustrate the proportion of lean patients (BMl $\leq 23 \mathrm{~kg} / \mathrm{m} 2$ ) with specific diagnosis. Among all 512 patients, $76.2 \%$ had no NAFLD and the remaining $23.8 \%$ had NAFLD, among which, $85.3 \%$ had NAFL and $14.8 \%$ had NASH. FLIP, fatty liver inhibition of progression; NAFLD, nonalcoholic fatty liver disease; NAFL, non-alcoholic fatty liver; NASH, non-alcoholic steatosis hepatitis.

Supplementary Figure $\mathbf{3}$ | (A) Bar chart to illustrate the proportion of fibrosis stage in HBV-infected patients with or without steatosis. $53.6 \%$ had significant fibrosis $(\mathrm{S} \geq 2)$ among patients without steatosis and $47.3 \%$ had significant fibrosis $(\mathrm{S} \geq 2)$ among patients with steatosis. (B) Bar chart to illustrate the proportion of fibrosis stage in HBV-infected patients with or without lobular inflammation. 27.9\% had significant fibrosis $(\mathrm{S} \geq 2)$ among patients without lobular inflammation and $52.7 \%$ had significant fibrosis ( $\mathrm{S} \geq 2$ ) among patients with lobular inflammation. (C) Bar chart to illustrate the proportion of fibrosis stage in HBV-infected patients with or without cytological ballooning. $41.5 \%$ had significant fibrosis $(S \geq 2)$ among patients without cytological ballooning and $68.9 \%$ had significant fibrosis $(S \geq 2)$ among patients with cytological ballooning.

Impact on Disease Severity and Treatment Response. Liver Int. 37, 542-551. doi: 10.1111/liv.13271

Cheng, J. Y., Wong, V. W., Tse, Y. K., Chim, A. M., Chan, H. L., and Wong, G. L. (2016). Metabolic Syndrome Increases Cardiovascular Events But Not Hepatic Events and Death in Patients With Chronic Hepatitis B. Hepatology 64, 15071517. doi: 10.1002/hep.28778

Choi, H. S. J., Brouwer, W. P., Zanjir, W. M. R., De Man, R. A., Feld, J. J., Hansen, B. E., et al. (2020). Nonalcoholic Steatohepatitis Is Associated With LiverRelated Outcomes and All-Cause Mortality in Chronic Hepatitis B. Hepatology 71, 539-548. doi: 10.1002/hep.30857

Consultation, W.H.O.E. (2004). Appropriate Body-Mass Index for Asian Populations and Its Implications for Policy and Intervention Strategies. Lancet 363, 157-163. doi: 10.1016/S0140-6736(03)15268-3

European Association for the Study of the Liver, Electronic Address, E.E.E., Clinical Practice Guideline, P., Chair, Representative, E.G.B. and Panel, Members (2021). EASL Clinical Practice Guidelines on Non-Invasive Tests for Evaluation of Liver Disease Severity and Prognosis - 2021 Update. J. Hepatol. 75, 659-689. doi: 10.1016/j.jhep.2021.05.025

European Association for the Study of the Liver, Electronic Address, E.E.E. and European Association for the Study of The, L. (2017). EASL 2017 Clinical Practice Guidelines on the Management of Hepatitis B Virus Infection. J. Hepatol. 67, 370-398. doi: 10.1016/j.jhep.2017.03.021 
Hui, R. W. H., Seto, W. K., Cheung, K. S., Mak, L. Y., Liu, K. S. H., Fung, J., et al. (2018). Inverse Relationship Between Hepatic Steatosis and Hepatitis B Viremia: Results of a Large Case-Control Study. J. Viral Hepat. 25, 97-104. doi: $10.1111 /$ jvh. 12766

Kim, K., Kim, K. H., Kim, H. H., and Cheong, J. (2008). Hepatitis B Virus X Protein Induces Lipogenic Transcription Factor SREBP1 and Fatty Acid Synthase Through the Activation of Nuclear Receptor LXRalpha. Biochem. J. 416, 219-230. doi: 10.1042/BJ20081336

Kim, K. H., Shin, H. J., Kim, K., Choi, H. M., Rhee, S. H., Moon, H. B., et al. (2007). Hepatitis B Virus X Protein Induces Hepatic Steatosis via Transcriptional Activation of SREBP1 and PPARgamma. Gastroenterology 132, 1955-1967. doi: 10.1053/j.gastro.2007.03.039

Loomba, R., and Sanyal, A. J. (2013). The Global NAFLD Epidemic. Nat. Rev. Gastroenterol. Hepatol. 10, 686-690. doi: 10.1038/nrgastro.2013.171

Machado, M. V., and Diehl, A. M. (2016). Pathogenesis of Nonalcoholic Steatohepatitis. Gastroenterology 150, 1769-1777. doi: 10.1053/j.gastro.2016.02.066

Mani, H., and Kleiner, D. E. (2009). Liver Biopsy Findings in Chronic Hepatitis B. Hepatology 49, S61-S71. doi: 10.1002/hep.22930

Ma, S. Y., Sun, K. S., Zhang, M., Zhou, X., Zheng, X. H., Tian, S. Y., et al. (2020). Disruption of Plin5 Degradation by CMA Causes Lipid Homeostasis Imbalance in NAFLD. Liver Int. 40, 2427-2438. doi: 10.1111/liv.14492

Nascimbeni, F., Bedossa, P., Fedchuk, L., Pais, R., Charlotte, F., Lebray, P., et al. (2020). Clinical Validation of the FLIP Algorithm and the SAF Score in Patients With Non-Alcoholic Fatty Liver Disease. J. Hepatol. 72, 828-838. doi: 10.1016/j.jhep.2019.12.008

Ott, J. J., Horn, J., Krause, G., and Mikolajczyk, R. T. (2017). Time Trends of Chronic HBV Infection Over Prior Decades - A Global Analysis. J. Hepatol. 66 (1), 48-54. doi: 10.1016/j.jhep.2016.08.013

Peng, D., Han, Y., Ding, H., and Wei, L. (2008). Hepatic Steatosis in Chronic Hepatitis B Patients Is Associated With Metabolic Factors More Than Viral Factors. J. Gastroenterol. Hepatol. 23, 1082-1088. doi: 10.1111/j.14401746.2008.05478.x

Polaris Observatory C. (2018). Global Prevalence, Treatment, and Prevention of Hepatitis B Virus Infection in 2016: A Modelling Study. Lancet. Gastroenterol. Hepatol. 3 (6), 383-403. doi: 10.1016/S2468-1253(18)30056-6

Scheuer, P. J. (1991). Classification of Chronic Viral Hepatitis: A Need for Reassessment. J. Hepatol. 13, 372-374. doi: 10.1016/0168-8278(91)90084-o

Shen, F., Mi, Y. Q., Xu, L., Liu, Y. G., Wang, X. Y., Pan, Q., et al. (2019). Moderate to Severe Hepatic Steatosis Leads to Overestimation of Liver Stiffness Measurement in Chronic Hepatitis B Patients Without Significant Fibrosis. Aliment Pharmacol. Ther. 50, 93-102. doi: 10.1111/apt.15298

Thomopoulos, K. C., Arvaniti, V., Tsamantas, A. C., Dimitropoulou, D., Gogos, C. A., Siagris, D., et al. (2006). Prevalence of Liver Steatosis in Patients With Chronic
Hepatitis B: A Study of Associated Factors and of Relationship With Fibrosis. Eur. J. Gastroenterol. Hepatol. 18, 233-237. doi: 10.1097/00042737-200603000-00002 Wong, G. L., Wong, V. W., Choi, P. C., Chan, A. W., Chim, A. M., Yiu, K. K., et al. (2009). Metabolic Syndrome Increases the Risk of Liver Cirrhosis in Chronic Hepatitis B. Gut 58, 111-117. doi: 10.1136/gut.2008.157735

Younossi, Z., Anstee, Q. M., Marietti, M., Hardy, T., Henry, L., Eslam, M., et al. (2018). Global Burden of NAFLD and NASH: Trends, Predictions, Risk Factors and Prevention. Nat. Rev. Gastroenterol. Hepatol. 15, 11-20. doi: 10.1038/nrgastro.2017.109

Younossi, Z. M., Koenig, A. B., Abdelatif, D., Fazel, Y., Henry, L., and Wymer, M. (2016). Global Epidemiology of Nonalcoholic Fatty Liver Disease-MetaAnalytic Assessment of Prevalence, Incidence, and Outcomes. Hepatology 64, 73-84. doi: 10.1002/hep.28431

Zhang, J., Lin, S., Jiang, D., Li, M., Chen, Y., Li, J., et al. (2020). Chronic Hepatitis B and Non-Alcoholic Fatty Liver Disease: Conspirators or Competitors? Liver Int. 40, 496-508. doi: 10.1111/liv.14369

Zheng, R. D., Xu, C. R., Jiang, L., Dou, A. X., Zhou, K., and Lu, L. G. (2010). Predictors of Hepatic Steatosis in HBeAg-Negative Chronic Hepatitis B Patients and Their Diagnostic Values in Hepatic Fibrosis. Int. J. Med. Sci. 7, 272-277. doi: 10.7150/ijms.7.272

Zhong, G. C., Wu, Y. L., Hao, F. B., Rao, X. W., Yuan, X. W., Zhao, Y., et al. (2018). Current But Not Past Hepatitis B Virus Infection Is Associated With a Decreased Risk of Nonalcoholic Fatty Liver Disease in the Chinese Population: A Case-Control Study With Propensity Score Analysis. J. Viral Hepats. 25, 842-852. doi: 10.1111/jvh.12878

Conflict of Interest: The authors declare that the research was conducted in the absence of any commercial or financial relationships that could be construed as a potential conflict of interest.

Publisher's Note: All claims expressed in this article are solely those of the authors and do not necessarily represent those of their affiliated organizations, or those of the publisher, the editors and the reviewers. Any product that may be evaluated in this article, or claim that may be made by its manufacturer, is not guaranteed or endorsed by the publisher.

Copyright (c) 2022 Huang, Gan, Lai, Wang, Guo, Sheng, Chen, Guo, Cai, Wang, Zhao, Cao and Xie. This is an open-access article distributed under the terms of the Creative Commons Attribution License (CC BY). The use, distribution or reproduction in other forums is permitted, provided the original author(s) and the copyright owner(s) are credited and that the original publication in this journal is cited, in accordance with accepted academic practice. No use, distribution or reproduction is permitted which does not comply with these terms. 